\section{Response to: 'Anti-Ro52 autoantibodies are associated with interstitial lung disease and more severe disease in patients with juvenile myositis' by Sabbagh et al}

We read with great interest the article by Sabbagh et al ${ }^{1}$ published in the Annals of the Rheumatic Diseases. We congratulate the authors for their accuracy and the exhaustive data presented, which clearly demonstrate the importance of anti-Ro52 autoantibodies in disease monitoring, management and prognosis in patients with juvenile myositis. We would like, however, to highlight some key points.

First, in this study, ${ }^{1}$ the patients with juvenile myositis including juvenile dermatomyositis (JDM), juvenile polymyositis (JPM) and juvenile connective tissue disease-myositis (JCTM) overlap are a highly heterogeneous population. In particular, JCTM added a great heterogeneity to the study population. In reality, there has been still controversy on considering CTM as a distinct entity or rather a complication related to other connective tissue disease itself. $^{2}{ }^{3}$ A recent study ${ }^{3}$ showed a distinct entity of CTM compared with PM and DM, featuring more extramuscular involvement and more severe infections. Especially, the prevalence of interstitial lung disease (ILD) in patients with CTM (48\%) was significantly higher than that in those with PM (35\%) and DM (30.8\%). The results of the study by Sabbagh et al indicated that the positive rate of anti-Ro52 autoantibodies in patients with JCTM was higher than that in those with PM and DM. However, the positive rate is also highly heterogeneous among patients with different JCTM, varying from 0 (juvenile systemic sclerosis) to $29 \%$ (juvenile idiopathic arthritis). ${ }^{1}$ Furthermore, in subgroup analyses of this study, there was a significant difference regarding the prevalence of ILD between patients with and without anti-Ro52 autoantibodies only in JDM group, but no difference in JPM or JCTM groups. As such, anti-Ro52 autoantibodies seemed to be only associated with JDM, but not with JPM or JCTM. In addition, we consider it necessary to validate the association between anti-Ro52 autoantibodies and ILD and other clinical features separately in DM patients by univariate and multivariate analyses, given too few numbers of patients with JCTM or PM. If so, the results will be more significant for clinical practice.

Second, in this study, ${ }^{1}$ length of follow-up, as one of confounding factors, was included in multivariate analyses using linear or logistic regression to test the independent association of anti-Ro52 autoantibodies with clinical features of juvenile myositis. Although it was not detailed enough about the time when various clinical features occurred (at illness onset or diagnosis or during follow-up), we presume that authors investigated the association between anti-Ro52 autoantibodies and clinical features at illness onset or diagnosis. In this case, length of follow-up may have little impact on the results and should not be used as a confounder. In contrast, delay to diagnosis should be considered. If so, we wonder whether the close association between anti-Ro52 autoantibodies and ILD or other clinical features has been remained.

Third, the number of anti-Ro52-positive patients is small and even. Single-digit in some subgroup analyses, which could lead to a low statistical power.

In conclusion, considering high heterogeneity of myositis, the association between anti-Ro52 autoantibodies and clinical features in patients with junile myositis needs to be discussed in more detail based on classification of myositis. The importance of anti-Ro52 autoantibodies in disease monitoring, management and prognosis in JDM patients deserves high attention, while the significance of these autoantibodies for JPM and JCTM has remained to be verified.

\section{Zaixing Yang, ${ }^{\oplus 1}$ Yan Liang ${ }^{2}$}

${ }^{1}$ Huangyan Hospital of Wenzhou Medical University, Taizhou First People's Hospital, Taizhou, China

${ }^{2}$ Departmentof Laboratory Diagnostics, ChangzhengHospital, Shanghai, China

Correspondence to Dr Zaixing Yang, Huangyan Hospital of Wenzhou Medical University, Taizhou First People's Hospital, Taizhou 318002, China; yangzaixingdiyi@163.com

Contributors $Z Y$ and $Y L$ wrote this correspondence.

Funding The authors have not declared a specific grant for this research from any funding agency in the public, commercial or not-for-profit sectors.

Competing interests None declared.

Patient consent for publication Not required.

Provenance and peer review Not commissioned; internally peer reviewed. (c) Author(s) (or their employer(s)) 2019. No commercial re-use. See rights and permissions. Published by BMJ.

$$
\text { Check for updates }
$$

To cite Yang Z, Liang Y. Ann Rheum Dis Epub ahead of print: [please include Day Month Year]. doi:10.1136/annrheumdis-2019-215678

Received 7 May 2019

Accepted 9 May 2019

Ann Rheum Dis 2019;0:1. doi:10.1136/annrheumdis-2019-215678

\section{REFERENCES}

1 Sabbagh S, Pinal-Fernandez I, Kishi T, et al. Anti-Ro52 autoantibodies are associated with interstitial lung disease and more severe disease in patients with juvenile myositis. Ann Rheum Dis 2019. doi:10.1136/annrheumdis-2018-215004 (Epub ahead of print 24 Apr 2019).

2 Colafrancesco S, Priori R, Valesini G. Inflammatory myopathies and overlap syndromes: update on histological and serological profile. Best Pract Res Clin Rheumatol 2015;29:810-25.

3 Nuño-Nuño L, Joven BE, Carreira PE, et al. Overlap myositis, a distinct entity beyond primary inflammatory myositis: a retrospective analysis of a large cohort from the REMICAM registry. Int J Rheum Dis 2019. doi:10.1111/1756-185X.13559 (Epub ahead of print 10 Apr 2019). 